ABUNDANCE OF BERYLLIUM AND BORON 


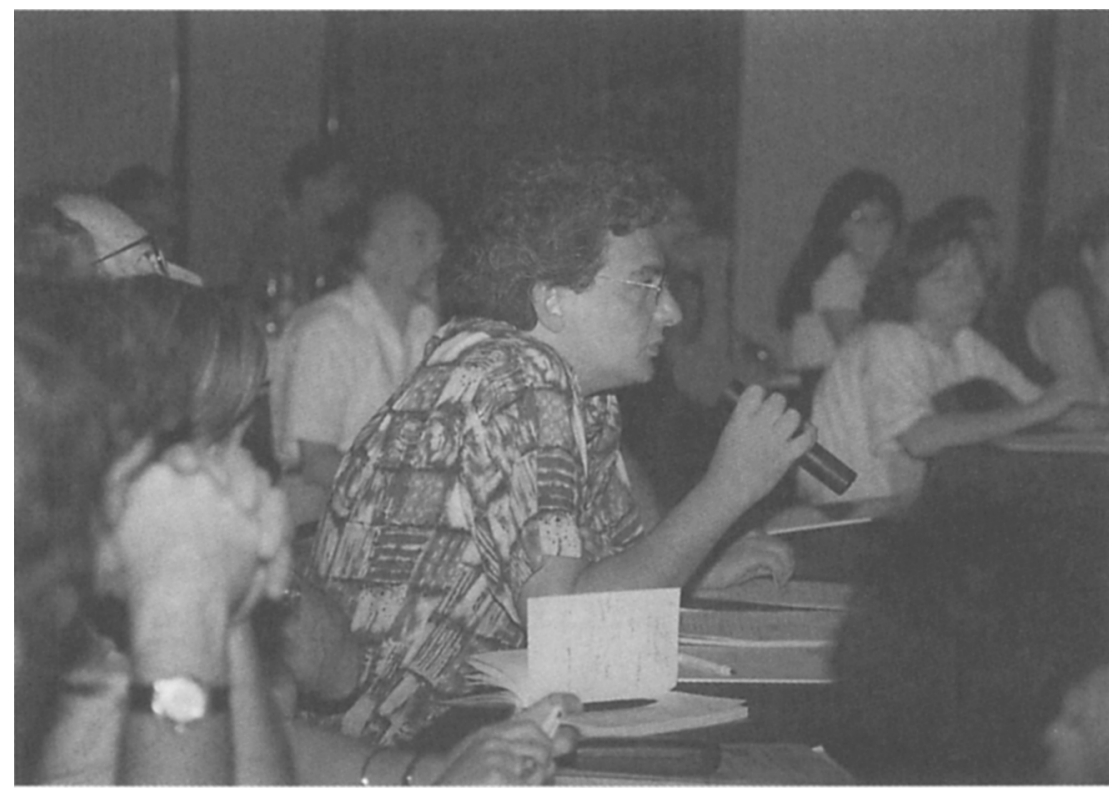

\section{Luca Pasquini}




\title{
Beryllium in the Sun: Re-Measurement and Implications
}

\author{
Suchitra C. Balachandran \\ Department of Astronomy, University of Maryland, College Park MD \\ 20742, USA
}

\begin{abstract}
The solar beryllium abundance is important because it provides a constraint on the depth to which mixing has occurred below the surface convective zone. Unlike helioseismology which only maps the present-day Sun, the solar beryllium abundance provides an integrated picture of mixing over the entire history of the Sun. In this review I outline the logic involving the "missing UV opacity" that required that the solar beryllium abundance be re-determined. A brief summary of the empirical process of estimating the "missing UV opacity" is given along with a confirmation based on a recent re-calculation of the Fe I boundfree opacity. The addition of this opacity resulted in our finding that the solar beryllium abundance was meteoritic. The implications of this result in the context of mixing in solar-type stars is discussed.
\end{abstract}

\section{Introduction}

The fragile element lithium, which is destroyed at temperatures greater than 2.5 million $\mathrm{K}$, is depleted by a factor of 140 in the solar photosphere relative to its meteoritic value. As the base of the solar convective zone is not hot enough to destroy lithium, this depletion requires surface material to be mixed to hotter temperatures below the convective zone. Such mixing is not predicted by the standard stellar models and the process which triggers the mixing is not understood. Non- standard stellar models (Pinsonneault et al. 1989; 1990; Charbonnel et al. 1994) have attempted to explain the depletion of lithium by linking it to a more ubiquitous process, the transport and dissipation of angular momentum. As a result of the contraction of the proto-stellar cloud, the young star is expected to be rotating rapidly when it arrives on the main sequence, and observations reveal that $G$ and $K$ stars in young clusters are often rapid rotators (see Stauffer 1991 for a review), in contrast to their older main sequence counterparts. It appears that at least a part of the angular momentum is lost while on the main sequence. The rotational models have suggested that the outer layers of the star are spun down initially by winds from the stellar surface, leaving the star in a state of differential rotation with radius. The transport of angular momentum is achieved via turbulence generated by shear forces between layers rotating at different rates . These models have predicted that a spread in lithium abundance will become apparent when stars with a range in initial angular momenta are spun down to the same final rotational velocity; a star with 
a larger initial angular momentum will undergo a greater amount of rotational spin-down, mixing and thus lithium depletion (Pinsonneault et al. 1990).

The principal interest in the solar photospheric abundance of beryllium (which is depleted at temperatures greater than 3.5 million $\mathrm{K}$ ) is to understand whether this more robust element has also been depleted relative to its meteoritic value. A depletion of lithium and beryllium would imply that mixing below the surface convective zone was more than merely superficial. The rotational models, which use the present-day solar lithium abundance as a constraint, predict that beryllium would be depleted by a factor of two. And indeed, this is precisely the result obtained by two recent measurements of the photospheric solar beryllium (Primas et al. 1997 and King et al. 1997).

In this review I will explain our re-measurement of the solar beryllium abundance, provide some clues for the opacity sources in the UV and discuss the implications of our result.

\section{The Opacity Problem and a Beryllium Re-Measurement}

Our re-measurement of the solar beryllium abundance was motivated by our recognition that the "UV Opacity Problem" would impact upon all abundance measurements from UV lines. The "UV Opacity Problem" is the mismatch between observed and predicted solar UV fluxes first recognized by Holweger (1970) and Gustafsson et al. (1975); theoretical UV fluxes are larger than observed leading to the surmise that the UV opacity is underestimated. After the inclusion of several million additional UV lines, Kurucz (1992) suggested that the "Opacity Problem" was a result of the insufficient inclusion of line opacity and demonstrated, at low resolution, that a match to the solar flux was achieved. However Bell et al. (1994) re-examined the problem at high resolution and found that many strong lines in Kurucz's line list were not present in the solar spectrum. They showed that a removal of these spurious lines resulted in a reversal back to the original problem: the predicted flux was too large. They concluded that the "missing opacity" was either a veil of very weak lines or a continuous opacity.

The implications of ignoring the "missing opacity" are the following. With insufficient opacity, the predicted line is stronger than observed, requiring the abundance to be decreased to match the observed line profile. An excellent example of this is the determination of boron abundances in Boesgaard et al. (1998); boron is measured from B I lines near $2500 \AA$. Stars undepleted in lithium were found to have boron abundances down by $0.7 \mathrm{dex}$ from the meteoritic value, a finding not explained by any mixing model. Recognizing this, Cunha et al. (2000) added Mg I bound-free opacity, the dominant opacity at this wavelength, to the analysis and found that stars undepleted in lithium were undepleted in boron as well. In reverse, if a solar oscillator strength is derived for a line, a spuriously large abundance will be calculated in, say, a metal-poor star in which contribution of the "missing opacity" may be negligibly small. The magnitude of the error will depend upon the magnitude of the "missing opacity". This logic formed the basis for our re-measurement of the solar beryllium abundance.

The impact of the "missing UV opacity" upon the solar beryllium abundance was recognized by Chmielewski et al. (1975). They determined that 
additional opacity had to be added to the synthetic fit to ensure that the spectrum of the center of the solar disk yielded the same abundance as that of the limb. They obtained a satisfactory but not complete match of the solar center and limb spectra and calculated $\log \epsilon(\mathrm{Be})=1.15 \pm 0.2$. Improvements in both spectral acquisition and opacity calculations since that study allow us to refine the opacity estimate.

The details of our re-measurement are given in Balachandran \& Bell (1998) and only a brief summary is provided here. The solar spectrum used was the digital version of the Kurucz et al. (1984) solar atlas. Three solar model atmospheres were used: the Holweger-Muller empirical model (Holweger \& Müller 1974), the OSMARCS (Edvardsson et al. 1993) and Kurucz (Kurucz 1993) theoretical models. With each model, the equivalent widths of the infrared $\mathrm{OH}$ lines from Grevesse et al. (1984) were analyzed to obtain the solar oxygen abundance. Fourteen clean OH lines were identified in the UV between 3100 and $3180 \AA$ and each of these were forced to yield the same oxygen abundance as the IR-based value obtained from that particular model atmosphere. Additional opacity was required to fit all of the lines. The mean additional opacity was taken as an estimate of the "missing opacity". The value of the "missing opacity" was the same from each of the models. When this additional opacity was used in the analysis of the Be II lines at $3130 \AA$ the beryllium abundance obtained was log $\epsilon(\mathrm{Be})=1.40 \pm 0.09$, essentially equal to the meteoritic value. The additional opacity required was an augmentation of the hydrogenic opacities by a factor of 1.6. However this did not provide a good fit to limb darkening; an augmentation of the Fe I bound-free opacity (from Dragon \& Mutschlecner 1980) of a factor of 30 provided both a better fit to the limb darkening and to the $\mathrm{OH}$ lines. The latter was chosen because of all the opacity sources which have some contribution at this wavelength, the Fe I bound-free opacity was the most uncertain.

We have recently been able to improve upon this empirical estimate of the "missing UV opacity" by including the revised Fe I bound-free opacity value of Bautista (1997) into our calculations. The details of this study are given in Bell, Balachandran \& Bautista (2000) and, again, only a brief summary is provided here. Bautista's Fe I bound- free opacity was included in our re-calculation of the continuous UV flux using the OSMARCS model. Observed line-blocking was added from the digital version of the Kurucz et al. (1984) solar atlas and a comparison of this UV flux was made to the SOLSTICE solar flux data from Woods et al. (1996). It was found that between 3000 and $4000 \AA$ a value of twice Bautista's (1997) opacity was required to obtain a good fit to the observed solar flux; this value is within the error limits set by Bautista for his calculations. The flux comparison was done at a very different resolution $(2.5 \AA)$ and over a much larger wavelength region $(3000-4000 \AA)$ than the OH-line fits $(0.008$ $\AA$ and 3100 - $3180 \AA$ respectively) that were used to obtain the earlier empirical estimate for the "missing opacity". We will shortly incorporate Bautista's Fe I b-f opacity into our stellar synthesis code and recalculate the opacity estimate via the $\mathrm{OH}$ lines. However, the flux fit is essentially compatible with our finding that the solar beryllium abundance is meteoritic; twice Bautista's Fe I b-f opacity is roughly equivalent to twenty times the Dragon \& Mutschlecner (1980) value. 


\section{Implications}

Two important predictions form the backbone of the angular momentum transport and material mixing via turbulence models. First, the models predict that the present-day Sun should have a rapidly rotating core. Helioseismological data have been interpreted to show that angular momentum transport and dissipation has been far more efficient than predicted by the turbulence transport models; the present-day Sun down to $\mathrm{r}=0.2 \mathrm{R}_{\odot}$ is rotating slowly as a rigid body (Tomczyk, Schou, \& Thompson 1995; Charbonneau et al. 1998). Alternatives to the transport of angular momentum via turbulence include magnetic fields (Charbonneau and MacGregor, 1993) and internal gravity waves (Kumar \& Quataert 1997; Zahn et al. 1997). Modeling of both forms of transport are still in their infancy, but the internal gravity wave models suggest that solid body rotation would be achieved in 10 Myr.

The second important prediction of the rotation models is that because differential rotation is expected to persist through the radiative interior for a large fraction of the star's main sequence lifetime, slow, deep mixing occurs. When constrained by the solar lithium abundance, this results in a depletion in beryllium by a factor of two. The lack of beryllium depletion in the solar photosphere, not just in the present-day Sun, but during its entire lifetime provides a very strong constraint on these non-standard stellar models. Helioseismic models of the present-day Sun indicate the presence of only a thin shear layer (the tachocline) at the base of the convective zone. Both the internal gravity wave model (Brun, Turck-Chièze, \& Zahn 1999) and the magnetic transport model (Barnes, Charbonneau \& MacGregor 1999) are able to reproduce the combination of lithium depletion by a factor of 140 and no beryllium depletion by invoking turbulence at the solar tachocline. Barnes et al. (1999) particularly point out that hydrodynamic transport of angular momentum results in both lithium and beryllium depletion while hydromagnetic transport reproduces the helioseismological data and lithium depletion while producing no beryllium depletion.

It appears, therefore, that the two strong bases for the transport of angular momentum and mixing via shear turbulence are no longer supported.

Modest beryllium depletion has been cited in three Hyades G dwarfs (García López et al. 1995) with beryllium decreasing by 0.2 dex between 5600 and 5200 $\mathrm{K}$. Given that the contribution of the Fe I b-f opacity increases with decreasing temperature, it is possible that this estimate may be due to the non-inclusion of sufficient Fe I bound-free opacity. Clearly additional calculations of the opacity contribution at different temperatures and a re-estimate of the beryllium abundance with its inclusion are crucial to determine if any depletion does occur in the $\mathrm{G}$ dwarfs.

The only dwarfs in which a real depletion of beryllium has been clearly measured are the F stars in the lithium 'dip' (Stephens et al. 1997; Deliyannis et al. 1998). It is clear that lithium is destroyed and not merely diffused out of sight in these stars (Balachandran 1995). Given the very shallow convective envelope and the relatively large lithium-preservation zones in these stars, the anomalous depletion of lithium requires considerable mixing below the surface convective zone. It is perhaps not surprising that such vigorous mixing is deep enough 
to result in beryllium depletion as well. The process by which the lithium-dip is produced is not understood, but the mixing mechanism may be completely disconnected from the process that causes lithium depletion in solar-type stars.

\section{References}

Balachandran, S. 1995, ApJ, 446, 203

Balachandran, S. C. \& Bell, R. A. 1998, Nature, 392, 791

Barnes, G., Charbonneau, P., \& MacGregor, K. B. 1999, ApJ, 511, 466

Bautista, M. 1997, A\&AS, 122, 167

Bell, R. A., Balachandran, S. C. \& Bautista, M. 2000, ApJL, submitted

Bell, R. A., Paltoglou, G., \& Tripicco, M. J. 1994, MNRAS, 268, 771

Boesgaard, A. M., Deliyannis, C. P., Stephens, A., \& Lambert, D. L. 1998, ApJ, 492, 727

Brun, A. S., Turck-Chièze, S., \& Zahn, J.-P. 1999, ApJ, 525, 1032

Charbonneau, P. \& MacGregor, K. B. 1993, ApJ, 417, 762

Charbonneau, P., Tomczyk, S., Schou, J., \& Thompson, M. J. 1998, ApJ, 496, 1015

Charbonnel, C., Vauclair, S., Maeder, A., Meynet, G., \& Schaller, G. 1994, A\&A, 283, 155

Chmielewski, Y., Brault, J. W., \& Müller, E. A. 1975, A\&A, 42, 37

Cunha, K., Smith, V. V., Boesgaard, A. M., \& Lambert, D. L. 2000, ApJ, 530, 939

Deliyannis, C. P., Boesgaard, A. M., Stephens, A., King, J. R., Vogt, S. S., \& Keane, M. J. 1998, ApJ, 498, L147

Dragon, J. N., \& Mutschlecner, J. P., 1980, ApJ, 239, 1045

Edvardsson, B. et al. 1993, A\&A, 275, 101

García López, R. J., Rebolo, R., \& Perez de Taoro, M. R. 1995, A\&A, 302, 184

Grevesse, N., Sauval, A. J., \& van Dischoeck, E. F. 1984, A\&A, 141, 10

Gustafsson, B., Bell, R. A., Eriksson, K., \& Nordlund, А., 42, 407

Holweger, H. 1970, A\&A, 4, 11

Holweger, H., \& Müller, E. A. 1974, Solar Phys. 39, 19

King, J. R., Deliyannis, C. P., \& Boesgaard, A., M., 1997, ApJ, 478, 778

Kumar, P., \& Quataert, E. J. 1997, ApJ, 473, L143

Kumar, P., Talon, A., \& Zahn, J.-P. 1999, ApJ, 520, 859

Kurucz, R. L. 1992, Rev. Mex. de Astromia y Astrofisica, 23, 181

Kurucz, R. L., 1993, CD Rom No. 13, SAO

Kurucz, R. L., Furenlid, I., Brault, J., \& Testerman, L. 1984, Solar Flux Atlas from 296 to 1200 nanometers (Natl. Solar Observatory, Tucson)

Pinsonneault, M. H., Kawaler, S. D., Sofia, S., \& Demarque, P. 1989, ApJ, 338, 424

Pinsonneault, M. H., Kawaler, S. D., \& Demarque, P. 1990, ApJS, 74, 501

Primas, F., Duncan, D. K., Pinsonneault, M. H., Deliyannis, C. P., \& Thorburn, J. A. 1997, ApJ, 480, 784

Stauffer, J. R. 1991, in Angular Momentum Evolution of Young Stars, eds. S. Catalano \& J. R. Stauffer, (Dordrecht: Kluwer), p. 117

Stephens, A., Boesgaard, A. M., King, J. R., \& Deliyannis, C. P. 1997, ApJ, 491,339 
Tomczyk, S., Schou, J., \& Thompson, M. J. 1995, ApJ, 448, L57

Woods, T. N. et al. 1996, JGR, D6, 9541

Zahn, J.-P., Talon, S., \& Matias, J. 1997, A\&A, 322, 320 